\title{
CONSTITUCION Y PODER TRIBUTARIO
}

Humberto Medrano C.

Una de las calidades inherentes al Estado es su capacidad para establecer tributos (Poder Tributario) cuyo pago constituye un deber de los contribuyentes. Sin embargo, este poder no puede ser ejercido en forma ilimitada, sin márgenes precisos de referencia, porque ello significaría dejar a los obligados a merced del Estado. Es por esta razón que la propia Carta Fundamental establece como garantía para los administrados, ciertos principios que regulan dicho poder y que configuran un marco sólo dentro del cual es posible exigir prestaciones tributarias.

\section{Principio de legalidad}

Sin duda es el más importante, tiene su origen en la Carta Magna arrancada a Juan Sin Tierra y por cuyo mérito los tributos sólo pueden establecerse con la aceptación de los llamados a pagarlos. En el Estado moderno ello equivale a que los tributos sólo pueden ser creados por el Poder Legislativo en tanto que sus miembros son representantes del pueblo $\mathrm{y}$, se asume, traducen su aceptación. Este principio puede sintetizarse con el aforismo "Nullum tributum sine lege": no hay tributo $\sin$ ley, de la misma manera en que no existe delito ni pena sin ley que asi lo determine.

La Constitución de 1933 en su artículo 80. señala "sólo para el servicio público podrá la ley crear, alterar o suprimir impuestos".

Este texto tiene obvias deficiencias ya que, en primer lugar, alude a impuestos, siendo así que éstos son sólo especies del género tributo. La clasificación más admitida divide el tributo en impuestos, contribuciones y tasas. (Véase, por ejemplo, el artículo 140. del modelo de Código Tributario para América Latina, preparado para el Programa Conjunto de Tributación OEA/BID.). La deficiencia constitucional anotada; trató de subsanarse a través del Código Tributario en cuyo artículo II del Título Preliminar se señala "el término genérico tributo incluye impuestos, contribuciones y tasas..." Efectuada tal definición el propio Código en su artículo IV recoge correctamente el principio de legalidad cuando indica: "Sólo por ley se puede crear, modificar o suprimir tributos..".

El artículo 139o. de la nueva Constitución establece: "Sólo por ley expresa se crean, modifican o suprimen tributos y se conceden exoneraciones y otros beneficios tributarios..." Este texto que, de suyo, consagra el principio resulta sin embargo reiterado, pues a continuación el mismo artículo señala "la tributación se rige por los principios de legalidad..."

Como se aprecia, la primera parte del artículo "contiene" el principio por lo que resulta innecesario denominarlo a continuación. Tratando de encontrar una respuesta a la reiteración podría sostenerse que la intención del legislador ha sido destacar en la primera parte la más importante limitación que se deriva del 
principio y que al insistir en denominarlo en su segunda parte se ha perseguido conseguir los alcances previstos en el resto del artículo IV del Título Prelininar del Código Tributario, de acuerdo con el cual sólo por ley se puede "...determinar el hecho imponible, fijar la cuantía del tributo y la base para su cálculo y señalar al contribuyente". Naturalmente la inclusión del precepto en el texto constitucional le otorga una permanencia y solidez que no tenia antes con su sola mención en el Código. No obstante, creemos que la reiteración era innecesaria tanto respecto de este principio como de otros que veremos más adelante.

Cabe mencionar que en nuestra jurisprudencia existen diversos casos en los que el Tribunal Fiscal ha puesto de relieve el principio de legalidad. Asi, "...los Concejos Municipales no están autorizados a cobrar arbitrios o tributos en general que no se establezcan por ley" (Resolución 7536 de 14 de setiembre de 1972). ".. el principio de legalidad domina el derecho tributario, principio consagrado por el artículo 8o. de la Constitución y por el artícuio IV del Título Preliminar del Código Tributario según los cuales sólo la ley puede crear tributos y conceder exoneraciones...". (Dictamen, Resolución 7304 de 4 de julio de 1972).

La nueva Carta (artículo 139o. tercer y cuarto párrafos) autoriza a los gobiemos regionales para crear, modificar, suprimir o exonerar tributos con arreglo a las facultades que se les delega por ley y a los gobiernos locales para crear, modificar y suprimir contribuciones, arbitrios o derechos o exonerar de ellos, conforme a ley.

El inciso 40. del artículo 254o. establece que las Municipalidades son competentes para crear, modificar o suprimir sus contribuciones, arbitrios y derechos, pero omite mencionar la sujeción a la ley. A pesar que la omisión nos parece sensible, debe interpretarse que esta facultad sólo puede ejercerse en caso de existir una ley autoritativa expresa, en razón de lo previsto en el artículo 1390.

Consideramos que la autorización a los gobiemos regionales y locales no debe conducir a los legisladores àl error de crear un tributo en forma genérica y facultar a los "delegatarios" para que éstos precisen el hecho imponible, la materia gravable o el sujeto pasivo, ya que ello no sólo no respetaría ei principio de legalidad sino que generaria los problemas que dicho principio trata de evitar. En efecto, los municipios siempre cortos de recursos, podrian apelar al fácil expediente de establecer tasas elevadas o aumentarlas cuando lo creyeran conveniente, con lo cual la garantía del contribuyente resultaría ilusoria.

Por otra parte, es evidente que la intención del legislador no ha sido dejar al arbitrio de los gobiemos regionales o locales la posiblidad de establecer los elementos esenciales de la relación jurídico-tributaria, porque para ello hubiera bastado que la Constitución les concediera lisa y llanamente el derecho de crear tributos, modificarlos, etc. Al haberse optado por conceder tales facultades con 
las restricciones que habrá de señalar la ley, resulta claro que lo que se persigue es que sea el Congreso quien establezca los marcos dentro de los cuales los otros organismos podrán ejercer tales facultades.

Debe quedar en claro que el poder tributario radica exclusivamente en el Estado y que los gobiernos regionales o locales tienen poder sólo en forma delegada, razón por la cual a través de la ley dicho poder puede ser reasumido integramente por el Estado en cualquier momento, En otras palabras, los gobiernos regionales y locales carecen de "soberania tributaria", en tanto que su poder no fluye directamente de la Constitución sino que ésta se limita a permitir que se le delegen ciertos atributos. En los paises dè organización federal el poder tributario de los diversos estados y sus limitaciones emanan de la Constitución, de forma tal que ellos sólo pueden ser modificados mediante reforma constitucional.

Nosotros consideramos que, habida cuenta de nuestra realidad, la ley autoritativa que se dicte tendrá que imponer marcos muy claros para evitar que, sin la garantía que significa la publicidad y discusión de un proyecto de ley, los entes menores puedan crear nuevos tributos o exonerar de ellos con criterios que, de alguna manera, pudieran resultar discriminatorios. Por tal razón, no creemos que el texto permita, por ejemplo, promulgar una ley estableciendo simplemente "autoricese a crear tributos" sino que el hecho imponible, el sujeto pasivo y la forma de establecer la cuantía del tributo deben quedar claramente fijados en la ley.

Por todo ello, sorprende que, en afán reglamentarista, el artículo 2570 . de la Constitución considere como rentas de las municipalidades los tributos que alli se mencionan, tales como las licencias y patentes, el el impuesto de rodaje, el impuesto a la extracción de materiales de construcción, etc. Hubiera sido más congruente que el texto estableciera que la ley señalara los tributos que constituyen renta municipal.

Obsérvese que el artículo se refiere a los tributos que gravan el valor de los predios urbanos y rústicos de su circunscripción. Con frecuencia los propietarios de predios urbanos son sociedades que no tributan sobre dichos bienes aisladamente considerados, sino como parte de sus activos que, naturalmente, comprenden muchos otros bienes. A raiz de este texto ¿deberán las empresas tributar al Gobiemo Central? ¿o lo que se pretendía era mantener como renta de los municipios sólo el impuesto al Patrimonio Predial No Empresarial, tal como establecen las normas vigentes? La redacción deficiente confirma que el detalle no debió establecerse constitucionalmente sino encargarse su especificación a una ley común.

Dicho artículo tal como está redactado tiene una consecuencia muy importante: Sólo las municipalidades pueden ingresar los tributos que se mencionan, de manera que ya no sería posible - ni siquiera por ley- dar el importe de los mismos destinos distinto, toda vez que el sujeto activo ha 
quedado establecido constitucionalmente y para variarlo sería necesaria una reforma constitucional. De otro lado, cabe preguntarse si la circunstancia de haberse establecido que determinados tributos constituyen renta de los municipios significa que tales tributos adquieren perpetuidad y no pueden ser modificados sin variar la Constitución. Nosotros creemos que ello no es así. Tal como hemos explicado, el poder tributario continúa residiendo en el Estado y por lo tanto una ley podría dejar sin efecto, modificar o ampliar el alcance de los tributos mencionados. En efecto, la Constitución no crea dichos tributos, sino establece que el rendimiento de los mismos es renta de las municipalidades.

En determinadas circunstancias podría convenir a la economía nacional derogar o modificar algunos de tales tributos, por lo que sería absurdo suponer que para eso se requiere de una reforma constitucional. Es nuestra opinión que tales tributos constituyen renta de los municipios en tanto ellos existan. Así por ejemplo, nuestra legislación no contempla el impuesto "a la extracción de materiales de construcción" mencionado en el inciso 6o. del artículo $257 \mathrm{o}$. La existencia de esta norma no faculta a las municipalidades para señalar al contribuyente o fijar la cuantía del impuesto, simplemente significa que tan pronto se dicte la ley formal que contenga todos los elementos de la relación tributaria, lo recaudado por ese impuesto debe constituir renta de lus municipalidades y sólo de ellas. Pero si el Parlamento no dicta la ley respectiva no puede entenderse que el tributo tenga vigencia.

Finalmente, el nuevo texto constitucional, al recoger el principio de legalidad, tiene el importante efecto de dejar sin valor el Decreto Ley 22168 en la parte que autoriza a modificar periódicamente por Decreto Supremo "la cuantía de los tributos denominados tasas que cobran las reparticiones del Gobierno Central, ...y las instituciones públicas". La misma suerte debe correr el Decreto Ley 21694 que autoriza al Poder Ejecutivo a señalar las tasas del impuesto a las revaluaciones.

El principio de legalidad se resiente de manera clara en el articulo 2110. inciso 22) que otorga al Presidente de la República la atribución de "regular las taridas arancelarias". Como quiera que dichas tarifas constituyen $-\sin$ dudatributos, su regulación sólo debería hacerse a través de la ley.

La excepción contenida en ese artículo se origina en el carácter complejo y especializado de la materia aduanera y en la fluidez con que, en ocasiones, deben manejarse las tarifas. Es decir, el constituyente ha optado por lo que entiende pragmático en desmedro del principio, decisión en la que -probablemente- han influído las circunstancias que el país vivía mientras se producía el debate.

La referida atribución significa que las decisiones del Presidente sobre la materia serán constitucionales, pero genera algunos interrogantes. En primer lugar, debe establecerse en que consiste "regular las tarifas..", pues si la aceptación correcta es la de "ajustar, reglar o poner en orden", ello supone la preexistencia de lo que es motivo de la regulación. Dentro de esta idea el 
Presidente podría modificar las tarifas del arancel pero no derogar o establecer el arancel mismo.

Sin embargo, nosotros creemos que el espíritu del texto ha sido dejar en manos del Presidente en forma exclusiva todo lo relativo a materia arancelaria, aún cuando la redacción no resulta muy feliz. En efecto, si la facultad hubiera estado referida - por ejemplo- a "regular" las tasas de los impuestos internos", no habria duda de que el principio de legalidad se mantendría respecto a la determinación de los sujetos, el hecho imponible, la oportunidad del pago, etc. y que sólo el "quantum" de tales tributos podráa ser establecido por el Presidente.

En cambio, en materia aduanera la regulación de tarifas constituye casi todo el universo legislativo, de modo que el inciso comentado implica que en este campo sólo el Presidente de la República tiene competencia. Por razones de claridad hubiera sido deseable que el artículo 1390 . señalara este caso como una excepción al principio.

Como quiera que la Constitución no establece el instrumento a través del cual el Presidente regulará las tarifas arancelarias, debemos asumir que deberá efectuarse mediante Decreto Supremo, lo cual plantea otra cuestión a considerar. Expedido el Decreto de Regulación ipodría el Congreso derogarlo o modificarlo a través de una ley? En razón de la jerarquía de las normas la respuesta debería ser, en principio, afirmativa, pero si se analiza con cuidado se observará que la atribución presidencial tiene su origen en la Constitución que, ciertamente, es de rango superior a la ley ordinaria.

Nosotros consideramos que el constituyente ha excluído del ámbito del Poder Legislativo todo lo referente a tarifas arancelarias, de forma tal que el Parlamento carece de competencia para pronunciarse sobre la materia. En tal virtud, las decisiones del Presidente no pueden ser modificadas por el Congreso, pues la ley que persiguiera ese propósito sería contraria a la Carta.

Por su parte, el artículo 1880. tiene también importancia respecto del principio pues permite "delegar en el Poder Ejecutivo la facultad de legislar mediante decretos legislativos sobre las materias y por el término que especifica la ley autoritativa". Obviamente, una de tales materias puede ser la tributaria, pero obsérvese que en este caso el principio de iegalidad se conserva de una foma que podría calificarse como "mediata", ya que el Congreso puede precisar el período de la delegación, los aspectos concretos a legislar y, en fin, los demás términos de referencia que estimara adecuados.

Aún más, los decretos legislativos pueden derogarse o modificarse por decisión del Congreso, de manera que -en última instancia-corresponde a éste aceptar o rechazar en todo o en parte, dichos decretos.

El artículo 188o. confirma que las decisiones del Presidente en materia arancelaria no pueden ser objetadas por el Parlamento. En efecto, este articulo 
hubiera permitido delegar en el Poder Ejecutivo la facultad de regular las tarifas arancelarias, lo que daria lugar a decretos legislativos derogables o modificables.

Si a pesar de existir esta norma el Constituyente ha optado por conferir al Presidente la atribución especifica de legislar en materia arancelaria, resulta claro que su intención ha sido otorgar esa potestad exclusivamente al Poder Ejecutivo privando de ella al Congreso. En otras palabras, la competencia del Presidente en este campo no supone necesidad de delegación por el legislativo, pues éste no puede delegar una facultad de la que carece.

\section{GENERALIDAD}

Uno de los elementos esenciales en la relación jurídico-tributaria es el "hecho imponible"; es decir, el supuesto previsto en la ley que en caso de ocurrir determina el surgimiento de la obligación. Producido el hecho imponible todas las personas comprendidas en su ámbito se encuentran obligadas.

Ciertamente hay algunos casos en los cuales no obstante haberse producido el supuesto legal, ciertos sujetos no están obligados a tributar en razón de exoneraciones o exclusiones del ámbito de aplicación del tributo. Estas excepciones no son violatorias del principio desde que -según se ha visto-el beneficio también debe ser otorgado por ley y en atención a valores que igualmente merecen protección constitucional. Así con arreglo a la nueva Carta: "El Estado.. concede exoneraciones tributarias a fin de abaratar la construcción" (artículo 180.) "Las universidades están exoneradas de todo tributo..." (Artículo 32o.)

En algunos casos estos beneficios se inspiran en el interés del Estado en desarrollar ciertas regiones del país (ley 15600 para la zona de la selva) la necesidad de alentar cierta clase de actividades económicas (Ley 16726 de Fomento Agropecuario).

En resumen, por el mérito de la "generalidad" todos los sujetos incluídos en el supuesto que la ley ha previsto están obligados a tributar. Las ex. clusiones (que sólo pueden otorgarse por ley) no violan el principio, en tanto se conceden a todos los que satisfacen los requisitos previstos por el legislador y en atención a valores que, la misma Constitución desea proteger.

Nuestra Constitución no utiliza el término "generalidaa" que es el que emplea la mayor parte de la doctrina, habiéndose preferido "uniformidad". En uno u otro caso cabe advertir que la denominación no era imprescindible, en tanto el principio fluye de otros artículos de la Constitución.

En efecto, el artículo 187 señala que "pueden expedirse leyes especiales porque lo exige la naturaleza de las cosas pero no por la dierencia de personas" y el segundo párrafo del ar. 139o. indica “... no hay privilegio personal en materia tributaria". 
Tales conceptos reflejan con claridad el principio, de modo que aludir a su denominación sólo puede tener su origen en el deseo del legislador de no dejar ninguna duda respecto de la existencia de esa garantía. La Constitución de 1933 tiene preceptos similares pues en su artículo 8o. establece "no hay privilegios personales en materia de impuestos", aún cuando debe observarse que por evidente deficiencia, sólo menciona impuestos. En este sentido el nuevo texto refleja mejor técnica pues alude a materia "tributaria" abarcando, así, con la expresión genérica, toda las especies.

Finalmente, cabe recordar que la constitución de 1933 en su artículo 230. también prohibe leyes especiales por la diferencia de personas.

\section{JUSTICIA}

Es obvio que se trata de una recomendación al legislador; corresponde a éste cuidar que al dictar la ley se afecte a cada quien de acuerdo a su capacidad contributiva; es decir, a su posibilidad económica de soportar el tributo. Se trata del principio doctrinario de la "igualdad" que no consiste en exigir lo mismo a todos, lo que sería injusto en una sociedad de grandes diferencias económicas, sino en hacer recaer el peso del tributo de manera pareja en todos los contribuyentes.

Ello supone medidas diferentes para diferentes capacidades económicas, de modo que todos soporten peso tributario de igual intensidad, aún cuando en términos cuantitativos la prestación sea distinta. Esto explica que se permitan mayores deducciones al casado que al soltero, al que tiene hijos respecto de quien no los tiene, etc. , Así por ejemplo, frente al "hecho imponible" de la percepción de una renta de igual monto, dos contribuyentes pueden pagat tributos de cuantía distinta en razón de diferentes capacidades contributivas. De acuerdo con este principio es razonable exigir los impuestos a la renta o al patrimonio no en función de una tasa proporcional, sino aplicando escalas progresivas y acumulativas.

$\mathrm{Si}$ se exigiera a todos contribuir con igual porcentaje de su renta $\mathrm{o}$ patrimonio se estaría atentando contra el principio, pues se impondría un sacrificio mayor a los de menor capacidad. También en este caso la denominación supone reiterar lo que ya aparece consignado en otros artículos del mismo texto. Así, conforme al artículo 77o. "todos tienen el deber de pagar los tributos que les corresponda y de soportar equitativanente las cargas establecidas por la ley para el sostenimiento de los servicios públicos".

Es evidente que cuando dicho artículo alude a "soportar equitativamente.', está efectuando una recomendación al legislador, por cuyo mérito éste debe tener presente que al dictar la norma sus consecuencias deben ser tales que produzcan una incidencia tributaria "homogénea", en el sentido aqui señalado, vale decir desigual entre los desiguales. 
La norma no permitiria a un contribuyente negarse a pagar un tributo argumentando que éste no resulta "equitativo" para él. Es el legislador quien debe tener en cuenta el precepto constitucional para tratar de aproximarse al ideal de justicia.

Debe observarse que la parte final del articulo 77o. se refiere a cargaś "establecidas para el sostenimiento de los servicios públicos".Aún cuando en esta parte del artículo no se utiliza la expresión tributo, cabe señalar que el sostenimiento de los servicios es un concepto superado, toda vez que con la tributación el Estado ya no persigue únicamente obtener parte de la riqueza de los particulares para poder prestar servicios, sino que ella tiene también importantes fines extrafiscales como proteger la industria nacional, desalentar el consumo de ciertos bienes, etc. Podemos asumir que al vincular la expresión "cargas" con los "servicios públicos" es probable que el constituyente se haya querido referir a las "tasas", pero como ya hemos dicho éstas no son sino una forma de tributo $\mathrm{y}$, por lo tanto, estarian comprendidas en la primera parte del artículo.

Defecto similar tiene al artículo 8o. de la Constitución de 1933 al señalar "sólo para el servicio público podrá la ley crear ...impuestos". Obśervese que que en este caso ni siquiera podría existir la explicación de referirse a las tasas sino que se menciona específicamente a los impuestos. La Constitución de 1933 no enuncia ningún principio, sino que ellos se encuentran implícitos en su articulado. Respecto del que comentamos, puede mencionarse que el artículo 23o. señala "la Constitución y las leyes protegen y obligan igualmente a todos los habitantes de la República"'

\section{PUBLICIDAD}

En este caso la reiteración es manifiestamente innecesaria por cuanto, de un lado, el propio artículo ya consagra el principio de legalidad y, por otra parte, el artículo 195o. establece que la ley es obligatoria después de su publicación en el Diario Oficial, aún cuando establece un precepto especial para ciertas leyes tributarias, a lo que nos referiremos más adelante.

Como quiera que la ley tributaria, desde la óptica que aquí interesa, es una ley como otra cualquiera no es posible percatarse de las razones que hayan llevado a establecer este principio, siendo así que el mismo ya aparece de otro artículo y es aplicable a toda clase de leyes. Podría intentarse una explicación señalando que la parte pertinente del artículo 139o. dice: "La tributación se rige por los principios de... publicidad..."; es decir no se expresa que la ley tributaria debe publicarse, lo que sería redundante en extremo, sino que "la tributación" debe hacerse de conocirriento público. En otras palabras todo lo relativo a la tributación y que no necesariamente es una ley debe ser materia de publicación. En este sentido debe recordarse que el artículo XI del Título Preliminar del Código Tributario indica que "las circulares que contengan directivas o instrucciones de carácter tributario que, por su naturaleza, sean de aplicación 
general, deberán ser también publicadas en el Diario Oficial, a partir de cuya fecha serán de observancia obligatoria para los contribuyentes". En consecuencia, podría inferirse que el principio de "publicidad" persigue dar rango constitucional a la citada norma del Código Tributario. Sin embargo creemos que se trata de una explicación insatisfactoria y estimamos que de haberse omitido este principio no se hubiera resentido la garantía que ya otorga el artículo 1950 .

La Constitución de 1933 no daba a las leyes tributarias un tratamiento especial, pues estaban incluídas en la norma general del articulo 1320. segíre el cual "la ley es obligatoria desde el día siguiente a su promulgación y publicación salvo disposición contraria de la misma ley".

\section{5.- OBLIGATORIEDAD}

Consideramos que su mención resulta innecesaria, pues todos los actos sustantivos en esta materia requieren ser expresados a través de una ley formal. La ley tributaria no es distinta a las demás y ella es ciertamente obligatoria para todos los que se encuentren incluídos en el hecho gravable previsto en la norma. En el ya citado artículo 195o. existe una fórmula general sobre la obligatoriedad de las leyes y, además, el artículo 77o. contiene un precepto especial sobre el deber de pagar tributos, de modo que la reiteración no tiene mayor significado.

\section{CERTEZA}

No debe entenderse sólo como una recomendación al legislador para que las normas que expida sean tan claras y precisas que no existan dudas sobre su sentido y alcances. Dicha recomendación sería deseable para todas las leyes y no sólo para las relativas a tributación. Por ello creemos que el articulo se refiere, en realidad, a la certeza que administradores y administrados deben tener respecto de sus derechos y obligaciones pero no sólo en función de una ley concreta sino de manera principista en toda relación jurídica originada por tributos. En otras palabras, la Carta confirma la necesidad de un cuerpo de leyes que, como el Código Tributario, establezca los principios generales, instituciones y normas del ordenamiento jurídico en este campo. Algunos de tales principios han asumido carácter constitucional otorgando, así, la mayor certeza posible en un Estado de Derecho.

\section{NO CONFISCATORIEDAD}

La doctrina ya habia considerado la no confiscatoriedad del tributo como uno de los límites del Poder Tributario, pero la vigencia del mismo se obtenía interpretando el artículo referente a la propiedad. Así, en la Constitución de 1933 , el artículo 290 . señala que "la propiedad es inviolable.. a nadie se puede privar de la suya, sino por causa de utilidad pública probada legalmente y previa indemnización justipreciada...". Es fácil percibir que esta garantía podría ser violada si por la vía del tributo se exige al contribuyente una prestación de cuantía tal que, en última instancia, suponga desplazar al Estado la totalidad o 
parte muy importante de la renta o patrimonio de los sujetos pasivos. En la nueva Constitución el artículo 125o. señala que a nadie puede privarse de su propiedad "sino por causa de necesidad y utilidad públicas o de interés social, declarada conforme a ley y previo pago en dinero de indemnización justipreciada.."”

Si bien ambos textos constitucionales permiten concluir que bajo la forma de tributo no puede llegarse a la confiscación, es evidente que para ello es necesario hacer una interpretación un tanto elíptica. La expresa prohibición del artículo 1390.: "no hay impuesto confiscatorio" establece de manera frontal una muy importante garantía, que persigue evitar que la propiedad se convierta en un derecho lírico. De no existir esta garantía el contribuyente estaría imposibilitado de oponerse a exigencias tributarias que, en el fondo, representen apoderamiento por el Estado de bienes de particulares sin pago alguno. Asi, en un ejemplo extremo, si el tributo sobre el patrimonio fuera igual al valor de éste, el contribuyente tendria que desprenderse del mismo para poder cumplir la obligación con lo cual -en la práctica- se estaría consumando simple y llanamente una confiscación.

Queda claro, en consecuencia, que el impuesto no puede representar para el contribuyente un egreso de monto tal que, en los hechos, implique desplazar hacia el Estado una parte tan significativa que le haga imposible restituir su patrimonio al nivel que tenia antes de pagar el tributo. Naturalmente, no es posible establecer a nivel constitucional cuál es la porción límite que puede tomar el Estado para no ser considerada confiscatoria.

Héctor Villegas explica que la Corte Suprema Argentina ha sostenido que los tributos son confiscatorios "cuando absorven una parte sustancial de la propiedad o de la renta" pero admite que hay dificultades para determinar correctamente que debe entenderse por "parte sustancial", para lo que no existe respuesta en términos absolutos, debiendo establecerse la razonabilidad de la imposición "en cada caso concreto según exigencias de tiempo y lugar". Compartimos totalmente esta opinión, pues corresponderá a los administradores invocar esta garantía frente a una ley específica, sin perjuicio de que la misma constituya un elemento de referencia para el legislador al dictar la norma. Villegas agrega que la Suprema Corte Argentina "ha fijado el 33o/o como tope de validez constitucional de ciertos tributos".

En resumen, consideramos que la "no confiscatoriedad" representa una importante garantía que coloca en este aspecto a nuestra Constitución como una de las más avanzadas. Sin embargo, cabe indicar que el texto prohibe el "impuesto" confiscatorio, siendo así que la prohibición debería comprender el "tributo" que tuviera esa conotación. En consecuencia, llegado el momento, respecto de tasas y contribuciones se tendria que esgrimir la norma que garantiza la propiedad. 
La circunstancia que el costo de recaudación de un tributo sea muy alto en relación con el ingreso que produce, no podría conducir a señalar que se trata de una ley "anticonstitucional", en el sentido de violación de garantías que la expresión supone. Por ello, creemos que no puede tener la calidad de "principio" de la tributación. Se trata simplemente de una recomendación más al legislador para no crear tributos que previsiblemente no habrán de procurar ingresos sustanciales o para derogar aquellos cuya vigencia no se justifica dentro de esa idea.

No obstante, creemos que en algunas circunstancias podría ser válido mantener la vigencia de un tributo determinado si con él no se persiguen fines fiscales (ingresos al Tesoro) sino propósitos extrafiscales como los que hemos citado en el punto 3 .

\section{IRRETROACTIVIDAD DE LA LEY TRIBUTARIA}

En el ámbito penal un acto sólo puede ser reprimible si en el momento de su comisión se encontraba expresamente tipificado como delito en una ley expresa. De la misma manera los "hechos imponibles" sólo son tales si al producirse están contemplados en la ley.

Lo expuesto supone que las leyes tributarias sólo pueden regir para el futuro excepto el caso de la retroactividad benigna donde surge nuevamente la similitud con el derecho penal. Sin embargo, cabe advertir que respecto de cierta clase de tributos no basta establecer que la ley debe regir para hechos que se produzcan despúśs de sur publieación, sino que su vigencia debe iniciarse en un futuro más o menos distante, pues de lo contrario se producirían efectos retroactivos, como explicamos más adelante.

De acuerdo con el artículo 187o. de la nueva Constitución "ningúna ley tiene fuerza ni efecto retroactivos, salvo en materia tributaria, cuando es más favorable al... contribuyente...". La retroactividad benigna en este campo no está contemplada en la Constitución de 1933, por lo que sólo cabe citar como antecedente el artículo 150 del Código Tributario, según el cual "la supresión o reducción de sanciones extinguirán o reducirán las que se encontraren en trámite o en ejecución".

Por otra parte el artículo 132 de la Constitución de 1933 precisa que "la ley es obligatoria desde el día siguiente de su promulgación y publicación...”, pero no contemplaba el caso especial de los tributos periódicos. Esta omisión trató de subsanarse mediante el artículo XI del Título Preliminar del Código Tributario, el cual establece que las leyes referidas "a tributos de periodicidad anual regirán desde el primer día del siguiente año calendario..." Es evidente que la intención del legislador es evitar que iniciado un período anual con la vigencia 
de determinadas leyes tributarias destinadas a afectar todo el ejercicio, ellas puedan ser modificadas en el curso del mismo. Considerando que normalmente el ejercicio económico se inicia el primero de enero y termina el 31 de diciembre, podría suceder que en el mes de noviembre de un año determinado se modificara el impuesto a la renta y se pretendiera que la modificación fuera aplicable a los resultados de ese ejercicio pronto a terminar. Obviamente, una modificación de esta clase impactaria negativamente toda vez que el factor tributario fue tomado como un costo por el contribuyente al iniciar el ejercicio, de manera que su alteración pocas semanas antes de concluir el año gravable afectaría las operaciones en forma irrecuperable en el mismo periodo. Este precepto, que evita consecurencias como la anotada, tiene ahora rango constitucional, pues según el artículo 195o. de la nueva Carta: "La ley es obligatoria desde el décimo sexto día ulterior a su publicación en el Diario Oficial... Las leyes que se refieren a tributos de periodicidad anual rigen desde el primer día del siguiente año calendario". La norma tiene importancia porque ya ha ocurrido que, a pesar de lo previsto en el Código, disposiciones legales referidas a tributos de periodicidad anual se han aplicado respecto de ejercicios iniciados. En este sentido la inclusión constitucional otorga permanencia a la garantía e impedirá que, como ya ha sucedido, una ley determinada señale que para ese caso concreto se deja en suspenso el artículo pertinente del Código Tributario (véase por ejemplo el Decreto Ley 17599 de 22 de abril de 1969). Esta actitud encontraba respaldo en la circunstancia de que siendo el código sólo una ley, ella podría ser modificada por otra norma de igual rango.

Debe anotarse, sin embargo, que ni el artículo XI del Título Preliminar del Código Tributario ni el artículo 195o. de la nueva Constitución solucionan el problema que se presenta en la práctica a las empresas que han sido autorizadas para cerrar sus balances en fechas distintas al 31 de diciembre. Imaginemos el caso de quienes están autorizados para que sus ejercicios corran desde el 10. de julio de un año al 30 de junio del año siguiente. Si la norma es promulgada en el mes de setiembre de un año (1), ella rige a partir del 1o. de enero del año siguiente (2), lo cual significa que cuando el contribuyente del ejemplo cierra su ejercicio en junio del año (2) ya se encuentra vigente la nueva norma, por lo que las exigencias de ésta le resultarían aplicables. Es nuestra opinión que el sentido del Código Tributario lleva a concluir que en el ejemplo propuesto la nueva norma kegal sólo sería aplicable para el ejercicio que se inicia el 10 . de julio del año (2) y no para el que se cerró el 30 de junio de ese año. Sin embargo, la Dirección General de Contribuciones estima que las normas legales sobre el impuesto a la renta publicadas en un año (1) son aplicables al ejercicio iniciado antes de la publicación, si tal ejercicio se cierra en cualquier momento del año (2).

En este sentido puede verse el Informe No. 499-79-EF/74-12 de 24 de octubre de 1979 , emitido por là mencionada Dirección y donde se sostiene que la modificación del Impuesto a la renta prevista en el Decreto Ley 22401 , que se publicó el 22 de diciembre de 1978 , debe aplicarse al ejercicio que se inició el 1o. de junio de 1978 y que concluye el 31 de mayo de 1979 . 
En un ejemplo extremo y siguiendo el criterio de la administración cabe ponerse en el caso de una empresa cuyo ejercicio gravable está comprendido entre el 1o. de febrero de un año (1) y el 31 de enero del siguiente (2). Si en el mes de diciembre del año (1) se aumentara considerablemente el impuesto a la renta, la ley tendría vigencia a partir del 1o. de enero del año (2), de manera que los resultados obtenidos al 31 de enero de ese año (2) se verían afectados con la nueva disposición legal. Es decir, que a pesar de haber transcurrido once de los doce meses del ejercicio sus resultados estarían incididos por la nueva ley, no obstante que el espíritu era, precisamente, evitar que se modifiquen los tributos periódicos vigentes el primer día del ejercicio gravable. En nuestra opinión hubiera sido deseable que la Constitución estableciera que tratándose de tributos de periodicidad anual las leyes rigen para el ejercicio que se inicie a partir del 10. de enero del año siguiente al de publicación.

En cualquier caso, debe quedar claro que el artículo 1950. de la Carta constituye adecuada garantía para la mayoría de contribuyentes, que son los que cierran sus ejercicios al 31 de diciembre de cada año. 